\title{
WEAK NONTRIVIAL SOLUTIONS OF DIRICHLET DISCRETE NONLINEAR PROBLEMS
}

\author{
R. SANOU ${ }^{1}$, B. $\mathrm{KONE}^{2, *}$ \\ ${ }^{1}$ Laboratoire de Mathématiques et Informatique (LAMI), UFR, Sciences Exactes et \\ Appliquées, Université Joseph-Ki-ZERBO, 03 BP 7021 Ouaga 03, Ouagadougou, Burkina-Faso \\ ${ }^{2}$ Laboratoire de Mathématiques et Informatique (LAMI), Institut Burkinabé des Arts et Métiers, Université \\ Joseph-Ki-ZERBO, 03 BP 7021 Ouaga 03, Ouagadougou, Burkina-Faso \\ *Corresponding author: leizon71@gmail.com \\ Received Jul. 7, 2020
}

\begin{abstract}
Авstract. In this paper we prove the existence of solutions to limit value problems. In the literature, these problems are taken as models in different fields of mathematical physics.

The main existence results are obtained by using a critical point theory and the Ekelands variational principle by applying the mountain pass geometry lemma.

2010 Mathematics Subject Classification. 47A75; 35B38; 35P30; 34L05; 34L30.

Key words and phrases: discrete boundary value problem; critical point; weak solution; mountain pass geometry lemma; Palais-Samale condition.
\end{abstract}

\section{INTRODUCTION}

Difference equations describe different phenomena in several fields such as economics, physics, mechanics, computer science and finance see for example $[1,5,12]$ and the references cited therein. Because of its importance in applications, there is a large literature about it. For example, based on the method of minimization, in [11], Koné and Ouaro proved the existence of a single weak solution to the following Dirichlet problem

$$
-\Delta(a(k-1, \Delta u(k-1)))=f(k), k \in \mathbb{N}[1, T],
$$

where $T \geq 2$ is a positive integer.

Also, in [14], based on critical point theory, Mihailescu and his collaborators proved the

DOI: 10.28924/APJM/7-33

(2020 Asia Pacific Journal of Mathematics 
existence of a continuous spectrum of eigenvalues for the Dirichlet problem

$$
-\Delta\left(|\Delta u(k-1)|^{p(k-1)-2} \Delta u(k-1)\right)=\lambda|u(k)|^{q(k)-2} u(k), k \in \mathbb{N}[1, T],
$$

where $T$ is a positive integer, $p: \mathbb{N}[0, T] \longrightarrow[2,+\infty)$ and $q: \mathbb{N}[1, T] \longrightarrow[2,+\infty)$ are bounded while $\lambda$ is positive constant.

M. Galewski and R. Wieteska studied in [7], by using variational methods and a critical point theory, the existence and the multiplicity of positive solutions for a perturbed anisotropic difference equation with dependence on a numerical parameter:

$$
\Delta\left(\alpha(k)|\Delta u(k-1)|^{p(k-1)-2} \Delta u(k-1)\right)+\lambda f(k, u(k))=0, k \in \mathbb{N}[1, T],
$$

with some function $\alpha$, a nonlinear term $\mathrm{f}$ and a numerical non negative parameter $\lambda$. The authors derived the intervals of a numerical parameter $\lambda$ for which the considered boundary value problem has at least one, exactly one, or at least two positive solutions. Some useful discrete inequalities are also derived.

In this paper, motivated by the above facts, we propose the following anisotropic nonlinear discrete Dirichlet problem

$$
\left\{\begin{array}{l}
-\Delta[\alpha(k-1) a(k-1, \Delta u(k-1))]=\lambda f(k, u(k)), k \in \mathbb{N}[1, T], \\
u(0)=u(T+1)=0,
\end{array}\right.
$$

where $\lambda>0$ is a numerical parameter. $\Delta u(k)=u(k+1)-u(k)$ is the forward difference operator, $\mathbb{N}[1, T]=\{1, \ldots, T\}$, with $T \geq 2, a, \alpha$ and $f$ are functions to be defined later.

Our paper is organized in the following way. In section 2 we define the general results that we will use throughout our work. In section 3, under suitable assumptions on the data, we show that problem (1.1) admits at least a weak nontrivial solution. This is subdivided into four subsections. In the first we use the minimization method; in the second we apply mountain pass geometry lemma, then in the third we apply the Ekelands variational principle and finally we managed to show that the problem (1.1) has at least two nontrivial weak solutions. In section 4 we proceed to an extension of the previous results.

\section{Preliminaries}

By a solution to problem (1.1) we mean such a function $u: \mathbb{N}[0, T+1] \longrightarrow \mathbb{R}$ which satisfies the given equation on $\mathbb{N}[1, T]$ and the boundary conditions. In the $T$-dimensional Hilbert 
space

$$
X=\{u: \mathbb{N}[0, T+1] \longrightarrow \mathbb{R}: u(0)=u(T+1)=0\},
$$

with the inner product

$$
\langle u, v\rangle=\sum_{k=1}^{T+1} \Delta u(k-1) \Delta v(k-1), \quad \forall u, v \in X,
$$

we consider the norm

$$
\|u\|=\left(\sum_{k=1}^{T+1}|\Delta u(k-1)|^{2}\right)^{\frac{1}{2}}
$$

Let the function

$$
p: \mathbb{N}[0, T] \longrightarrow[2,+\infty)
$$

and denoted by

$$
p^{-}=\min _{k \in \mathbb{N}[0, T]} p(k), \quad p^{+}=\max _{k \in \mathbb{N}[0, T]} p(k) .
$$

For the data $\alpha, a$ and $f$, we assume the following.

$$
\left(H_{1}\right):\left\{\begin{array}{l}
a(k, .): \mathbb{R} \rightarrow \mathbb{R}, k \in \mathbb{N}[0, T] \text { and there exists } A(., .): \mathbb{N}[0, T] \times \mathbb{R} \rightarrow \mathbb{R} \\
\text { which satisfies } a(k, \xi)=\frac{\partial}{\partial \xi} A(k, \xi) \text { and } A(k, 0)=0, \text { for all } k \in \mathbb{N}[0, T] .
\end{array}\right.
$$

$\left(H_{2}\right):$ For all $k \in \mathbb{N}[0, T]$ and $\xi \neq \eta$

$$
(a(k, \xi)-a(k, \eta)) \cdot(\xi-\eta)>0
$$

$\left(H_{3}\right):$ For any $k \in \mathbb{N}[0, T], \xi \in \mathbb{R}$, we have

$$
p(k) A(k, \xi) \geq a(k, \xi) \xi \geq|\xi|^{p(k)}
$$

$\left(H_{4}\right):$ For any $k \in \mathbb{N}[0, T], \xi \in \mathbb{R}$ it exist $C_{1}>0$ such that

$$
|a(k, \xi)| \leq C_{1}\left(1+|\xi|^{p(k)-1}\right)
$$

$\left(H_{5}\right)$ : The function $\alpha: \mathbb{N}[0, T] \longrightarrow(0,+\infty)$ is such that for all $k \in \mathbb{N}[0, T]$,

$$
0<\underline{\alpha}=\inf _{k \in \mathbb{N}[0, T]}(\alpha(k)) \leq \alpha(k) \leq \bar{\alpha}=\sup _{k \in \mathbb{N}[0, T]}(\alpha(k))<+\infty .
$$

$\left(H_{6}\right):$ For each $k \in \mathbb{N}[1, T]$, the function $f(k,):. \mathbb{R} \longrightarrow \mathbb{R}$ is jointly continuous and there exist the functions $A_{1}, A_{2}: \mathbb{N}[1, T] \longrightarrow(-\infty, 0) ; B_{1}, B_{2}: \mathbb{N}[1, T] \longrightarrow(0,+\infty)$ and a function $r: \mathbb{N}[1, T] \longrightarrow[2,+\infty)$ such that

$$
A_{1}(k)+B_{1}(k)|\xi|^{r(k)-1} \leq f(k, \xi) \leq A_{2}(k)+B_{2}(k)|\xi|^{r(k)-1},
$$


where

$$
\begin{aligned}
& -\infty<\underline{A_{1}}=\inf _{k \in \mathbb{N}[1, T]} A_{1}(k), \quad \overline{A_{1}}=\sup _{k \in \mathbb{N}[1, T]} A_{1}(k)<0 ; \\
& -\infty<\underline{A_{2}}=\inf _{k \in \mathbb{N}[1, T]} A_{2}(k), \quad \overline{A_{2}}=\sup _{k \in \mathbb{N}[1, T]} A_{2}(k)<0 ; \\
& 0<\underline{B_{1}}=\inf _{k \in \mathbb{N}[1, T]} B_{1}(k), \quad \overline{B_{1}}=\sup _{k \in \mathbb{N}[1, T]} B_{1}(k)<+\infty ; \\
& 0<\underline{B_{2}}=\inf _{k \in \mathbb{N}[1, T]} B_{2}(k), \quad \overline{B_{2}}=\sup _{k \in \mathbb{N}[1, T]} B_{2}(k)<+\infty ; \\
& r^{-}=\min _{k \in \mathbb{N}[1, T]} r(k), \quad r^{+}=\max _{k \in \mathbb{N}[1, T]} r(k)
\end{aligned}
$$

and there exists

$$
\beta_{1}, \beta_{2}>1 \text { such that } \underline{B_{1}}>\max \left(\beta_{1}, \beta_{2}\right)\left|\underline{A_{1}}\right| r^{+} \text {. }
$$

We denote

$$
F(k, \xi)=\int_{0}^{\xi} f(k, s) d s \text { for }(k, \xi) \in \mathbb{N}[0, T] \times \mathbb{R} .
$$

There are many functions satisfying both $\left(H_{1}\right)-\left(H_{6}\right)$. Let us mention the following.

\section{Example 2.1.}

- $A(k, \xi)=\frac{1}{p(k)}\left(\left(1+|\xi|^{2}\right)^{p(k) / 2}-1\right)$ with $a(k, \xi)=\left(1+|\xi|^{2}\right)^{(p(k)-2) / 2} \xi, \forall k \in \mathbb{N}[0, T], \forall \xi \in \mathbb{R}$,

- $f(k, \xi)=-1+|\xi|^{r(k)-1}, \forall k \in \mathbb{N}[1, T], \quad \forall \xi \in \mathbb{R}$,

- $\alpha(k)=1, \forall k \in \mathbb{N}[0, T]$.

Moreover, we may consider $X$ with the following norm

$$
|u|_{m}=\left(\sum_{k=1}^{T}|u(k)|^{m}\right)^{\frac{1}{m}}, \quad \forall u \in X \quad \text { and } \quad m \geq 2 .
$$

We have the following inequalities (see [3])

$$
T^{(2-m) /(2 m)}|u|_{2} \leq|u|_{m} \leq T^{1 / m}|u|_{2}, \quad \forall u \in X \quad \text { and } \quad m \geq 2 .
$$

We need the following auxiliary results throughout our paper (see $[7,8,14])$. 


\section{Lemma 2.1.}

(1) There exist two positive constants $C_{2}, C_{3}$ such that

$$
\sum_{k=1}^{T+1}|\Delta u(k-1)|^{p(k-1)} \geq C_{2}\|u\|^{p^{-}}-C_{3}
$$

for all $u \in X$ with $\|u\|>1$.

(2) For every $u \in X$ with $\|u\| \leq 1$ we have

$$
\sum_{k=1}^{T+1}|\Delta u(k-1)|^{p(k-1)} \geq T^{-\frac{p^{+}-2}{2}}\|u\|^{p^{+}} .
$$

(3) For any $m \geq 2$ there exists a positive constant $c_{m}$ such that

$$
\sum_{k=1}^{T}|u(k)|^{m} \leq c_{m} \sum_{k=1}^{T+1}|\Delta u(k-1)|^{m}, \quad \forall u \in X .
$$

(4) For every $u \in X$ we have

$$
\sum_{k=1}^{T+1}|\Delta u(k-1)|^{p(k-1)} \leq(T+1)\|u\|^{p^{+}}+(T+1) .
$$

(5) For every $u \in X$ and for every $m \geq 2$ we have

$$
\sum_{k=1}^{T+1}|\Delta u(k-1)|^{m} \leq 2^{m} \sum_{k=1}^{T}|u(k)|^{m} .
$$

Theorem 2.1. [13] Let $H$ be a reflexive Banach space. If a functional $J \in C^{1}(H, \mathbb{R})$ is weakly lower semi-continuous and coercive, i.e. $\lim _{\|x\| \rightarrow \infty} J(x)=+\infty$, then there exists $x_{0}$ such that

$$
\inf _{x \in H} J(x)=J\left(x_{0}\right)
$$

and $x_{0}$ is also a critical point of $J$, i.e. $J^{\prime}\left(x_{0}\right)=0$. Moreover, if $J$ is strictly convex, then a critical point is unique.

Theorem 2.2. [6](Ekeland's principle) Let E be a complete metric space and $\Phi: E \longrightarrow \mathbb{R}$ a lower semicontinuous function that is bounded below. Let $\varepsilon>0$ and $\bar{u} \in E$ be given such that

$$
\Phi(\bar{u}) \leq \inf _{E} \Phi+\frac{\varepsilon}{2}
$$

Then given $\lambda>0$ there exists $u_{\lambda} \in E$ such that

(i) $\Phi\left(u_{\lambda}\right) \leq \Phi(\bar{u})$,

(ii) $d\left(u_{\lambda}, \bar{u}\right)<\lambda$,

(iii) $\Phi\left(u_{\lambda}\right)<\Phi(u)+\frac{\varepsilon}{\lambda} d\left(u, u_{\lambda}\right)$ for all $u \neq u_{\lambda}$. 
Definition 2.1. Let $H$ be a real Banach space. We say that a functional $J: H \longrightarrow \mathbb{R}$ satisfies the Palais-Smale condition if every sequence $\left(u_{n}\right)$ such that $\left\{J\left(u_{n}\right)\right\}$ is bounded and $J^{\prime}\left(u_{n}\right) \rightarrow 0$ has a convergent subsequence.

Lemma 2.2. [4] Let $H$ be a Banach space and $J \in C^{1}(H, \mathbb{R})$ satisfies the Palais-Smale condition. Assume that there exist $x_{0}, x_{1} \in H$ and a bounded open neighborhood $\Omega$ of $x_{0}$ such that $x_{1} \notin \bar{\Omega}$ and

$$
\max \left\{J\left(x_{0}\right), J\left(x_{1}\right)\right\}<\inf _{x \in \partial \Omega} J(x)
$$

Let

$$
\Gamma=\left\{h \in C([0,1], H): h(0)=x_{0}, \quad h(1)=x_{1}\right\}
$$

and

$$
c=\inf _{h \in \Gamma} \max _{s \in[0,1]} J(h(s)) .
$$

Then $c$ is a critical value of $J$; that is, there exists $x^{*} \in H$ such that $J^{\prime}\left(x^{*}\right)=0$ and $J\left(x^{*}\right)=c$, where $c>\max \left\{J\left(x_{0}\right), J\left(x_{1}\right)\right\}$.

Theorem 2.3. [2] Let E be a finite-dimensional Euclidean space, $\eta, \mu_{1}, \mu_{2}: E \longrightarrow \mathbb{R}$ be differentiable functions, and $S=\left\{x \in E: \mu_{1} \leq 0, \mu_{2} \leq 0\right\}$. Moreover, let $\bar{x} \in S$ be such that $\eta(\bar{x})=\inf _{S} \eta(x)$. Then there exist numbers $\sigma_{0}, \sigma_{1}, \sigma_{2} \geq 0$ such that $\left(\sigma_{0}\right)^{2}+\left(\sigma_{1}\right)^{2}+\left(\sigma_{2}\right)^{2}>0$ and

$$
\sigma_{0} \eta^{\prime}(\bar{x})+\sigma_{1} \mu_{1}^{\prime}(\bar{x})+\sigma_{2} \mu_{2}^{\prime}(\bar{x})=0 \quad \text { and } \quad \sigma_{1} \mu_{1}(\bar{x})=0, \quad \sigma_{2} \mu_{2}(\bar{x})=0
$$

\section{Existence of SOlutions}

In this section, we will prove the existence of at least one weak nontrivial solution to the problem (1.1).

This section is divided into four subsections.

We define the energy functional corresponding to $(1.1) ; J_{\lambda}: X \longrightarrow \mathbb{R}$ by

$$
J_{\lambda}(u)=\sum_{k=1}^{T+1} \alpha(k-1) A(k-1, \Delta u(k-1))-\lambda \sum_{k=1}^{T} F(k, u(k)) .
$$

The functional $J_{\lambda}$ is differentiable in sense of Gâteaux and its Gâteaux derivative reads

$$
\left\langle J_{\lambda}^{\prime}(u), v\right\rangle=\sum_{k=1}^{T+1} \alpha(k-1) a(k-1, \Delta u(k-1)) \Delta v(k-1)-\lambda \sum_{k=1}^{T} f(k, u(k)) v(k),
$$


for all $u, v \in X$.

A critical point to $J_{\lambda}$, i.e. a point $u \in X$ such that

$$
\left\langle J_{\lambda}^{\prime}(u), v\right\rangle=0 \quad \text { for all } \quad v \in X
$$

is a weak solution to (1.1). The previous results have been proven in $[9,10,15]$.

Lemma 3.1. Assume that (2.5), (2.7) holds and $r^{-}>p^{+}$. Then for any $\lambda>0$ the functional $J_{\lambda}$ satisfies the Palais-Smale condition.

Proof Assume that $\left\{u_{n}\right\}$ is such that $\left\{J_{\lambda}\left(u_{n}\right)\right\}$ is bounded and $J_{\lambda}^{\prime}\left(u_{n}\right) \rightarrow 0$. We have $X$ finitely dimensional, it is enough to show that $\left\{u_{n}\right\}$ is bounded. Assume that $\left\{u_{n}\right\}$ is unbounded, we have, for $n$ large enough, $\left\|u_{n}\right\|$ sufficiently large. We consider $n$ large enough and by (2.5), (2.7), (2.10), (2.14), (2.15) we have

$$
\begin{aligned}
& J_{\lambda}\left(u_{n}\right) \leq \bar{\alpha} \sum_{k=1}^{T+1} \int_{0}^{\Delta u_{n}(k-1)}|a(k-1, s)| d s-\lambda\left(-\left|\underline{A_{1}}\right| \sum_{k=1}^{T}\left|u_{n}(k)\right|+\frac{B_{1}}{r^{+}} \sum_{k=1}^{T}\left|u_{n}(k)\right|^{r(k)}\right) \\
& \leq C_{1} \bar{\alpha} \sum_{k=1}^{T+1}\left(\left|\Delta u_{n}(k-1)\right|+\frac{\left|\Delta u_{n}(k-1)\right|^{p(k-1)}}{p^{-}}\right) \\
& -\lambda\left(-\left|\underline{A_{1}}\right| \sqrt{T} \sum_{k=1}^{T}\left|u_{n}(k)\right|^{2}+\frac{B_{1}}{\overline{r^{+}}} \sum_{k=1}^{T}\left|u_{n}(k)\right|^{r^{-}}\right) \\
& \leq C_{1} \bar{\alpha}\left(\sqrt{T+1}:\left\|u_{n}\right\|+\frac{(T+1)\left\|u_{n}\right\|^{p^{+}}}{p^{-}}+\frac{T+1}{p^{-}}\right) \\
& -\lambda\left(-\underline{\underline{A_{1}}} \mid \sqrt{T} c_{2}\left\|u_{n}\right\|^{2}+\frac{2^{-r^{-}}(T+1)^{\frac{2-r^{-}}{2}} \underline{B_{1}}}{r^{+}}\left\|u_{n}\right\|^{r^{-}}\right) .
\end{aligned}
$$

Since $r^{-}>p^{+}$and $\left\|u_{n}\right\| \rightarrow+\infty$; we have $J_{\lambda}\left(u_{n}\right) \rightarrow-\infty$. This is contradictory. Hence the sequence $\left\{u_{n}\right\}$ is bounded.

\subsection{Case $p^{-}>r^{+}$.}

In this subsection we will show that the problem (1.1) admits at least one weak nontrivial solution using the minimisation method.

Proposition 3.1. Assume that $(2.4),(2.7)$ holds and $p^{-}>r^{+}$, then $J_{\lambda}$ is coercive for all $\lambda>0$. 
Proof according to (2.4) and (2.7), we have

$$
\begin{aligned}
J_{\lambda}(u) & =\sum_{k=1}^{T+1} \alpha(k-1) A(k-1, \Delta u(k-1))-\lambda \sum_{k=1}^{T} F(k, u(k)) \\
& \geq \frac{\underline{\alpha}}{p^{+}} \sum_{k=1}^{T+1}|\Delta u(k-1)|^{p(k-1)}-\lambda\left(\frac{\overline{B_{2}}}{r^{-}} \sum_{k=1}^{T}|u(k)|^{r(k)}+\left|\underline{A_{2}}\right| \sum_{k=1}^{T}|u(k)|\right) .
\end{aligned}
$$

To prove the coerciveness of the functional $J_{\lambda}$, we consider $\|u\|>1$, and from the above inequalities (2.10), (2.11) and (2.13) we deduce that

$$
\begin{aligned}
J_{\lambda}(u) & \geq \frac{C_{2} \underline{\alpha}}{p^{+}}\|u\|^{p^{-}}-\frac{\underline{\alpha}}{p^{+}} C_{3}-\lambda\left(\frac{\overline{B_{2}}}{r^{-}} \sum_{k=1}^{T}|u(k)|^{r^{+}}+\frac{\overline{B_{2}}}{r^{-}} \sum_{k=1}^{T}|u(k)|^{r^{-}}+\left|\underline{A_{2}}\right| \sqrt{T} \sum_{k=1}^{T}|u(k)|^{2}\right) \\
& \geq \frac{C_{2} \underline{\alpha}}{p^{+}}\|u\|^{p^{-}}-\frac{\underline{\alpha}}{p^{+}} C_{3} \\
& -\lambda\left(\frac{\overline{B_{2}}}{r^{-}} c_{r^{+}} \sum_{k=1}^{T+1}|\Delta u(k-1)|^{r^{+}}+\frac{\overline{B_{2}}}{r^{-}} c_{r^{-}} \sum_{k=1}^{T+1}|\Delta u(k-1)|^{r^{-}}+\left|\underline{A_{2}}\right| \sqrt{T} c_{2} \sum_{k=1}^{T+1}|\Delta u(k-1)|^{2}\right) \\
& \geq \frac{C_{2} \underline{\alpha}}{p^{+}}\|u\|^{p^{-}}-\frac{\underline{\alpha}}{p^{+}} C_{3}-\lambda\left(\frac{\overline{B_{2}}}{r^{-}} c_{r^{+}}(T+1)\left|u\left\|^{r^{+}+\frac{\overline{B_{2}}}{r^{-}}} c_{r^{-}}(T+1)\right\| u\left\|^{r^{-}}+\left|\underline{A_{2}}\right| \sqrt{T} c_{2}\right\| u \|^{2}\right) .\right.
\end{aligned}
$$

Hence $p^{-}>r^{+}, J_{\lambda}$ is coercive.

Theorem 3.1. Assume that (2.5) and (2.7) holds and $p^{-}>r^{+}$. Then, there exists $\lambda_{0} \in(0,+\infty)$ such that for all $\lambda>\lambda_{0}$ the problem (1.1) has at least one weak nontrivial solution.

Proof. As in [11], $J_{\lambda} \in C^{1}(X, \mathbb{R})$ and weakly lower semicontinuous. Moreover by Proposition 3.1 we prove the Theorem 2.1. Let $u_{\varepsilon} \in X$ a global minimizer of $J_{\lambda}$, a weak solution of problem (1.1). We show $u_{\varepsilon}$ is not trivial for all $p^{-}>r^{+}$and $\lambda>\lambda_{0}$.

For $t_{0}>1$ be a fixed real and $k_{0} \in \mathbb{N}[0, T+1]$, we define $u_{0}: \mathbb{N}[0, T+1] \longrightarrow \mathbb{R}$ such that $u_{0}\left(k_{0}\right)=t_{0}$ and $u_{0}(k)=0$ for any $k \in \mathbb{N}[0, T+1] \backslash\left\{k_{0}\right\}$, we have $u_{0} \in X$. By (2.5) and (2.7)

$$
\begin{aligned}
J_{\lambda}\left(u_{0}\right) & \leq C_{1} \bar{\alpha}\left(2 t_{0}+\frac{t_{0}^{p\left(k_{0}-1\right)}+t_{0}^{p\left(k_{0}\right)}}{p^{-}}\right)-\lambda\left(-\left|\underline{A_{1}}\right| t_{0}+\frac{B_{1}}{r^{+}} t_{0}^{r^{-}}\right) \\
& \leq 4 C_{1} \bar{\alpha} t_{0}^{p^{+}}-\lambda t_{0}\left(-\left|\underline{A_{1}}\right|+\frac{B_{1}}{r^{+}}\right) \\
& \leq 4 C_{1} \bar{\alpha} t_{0}^{p^{+}}-\lambda t_{0}\left(-\max \left(\beta_{1}, \beta_{2}\right)\left|\underline{A_{1}}\right|+\frac{\underline{B_{1}}}{r^{+}}\right)
\end{aligned}
$$

where

$$
\lambda_{0}=\frac{4 C_{1} \bar{\alpha} t_{0}^{p^{+}-1}}{-\max \left(\beta_{1}, \beta_{2}\right)\left|\underline{A_{1}}\right|+\frac{B_{1}}{r^{+}}} .
$$


We have $J_{\lambda}\left(u_{0}\right)<0$ for any $\lambda \in\left(\lambda_{0},+\infty\right)$.

It follows that $J_{\lambda}\left(u_{\varepsilon}\right)<0$ for any $\lambda>\lambda_{0}, u_{\varepsilon}$ is a weak nontrivial solution of problem (1.1) for $\lambda$ large enough.

\subsection{Case $r^{-}>p^{+}$.}

We have previously shown that if $r^{-}>p^{+} J_{\lambda}$ satisfies the Palais-Smale condition. We can thus use the mountain pass geometry Lemma.

Theorem 3.2. Let $r^{-}>p^{+}$and the condition (2.7) holds. Then, there exists $\lambda_{1} \in(0,+\infty)$ such that for $\lambda \in\left(0, \lambda_{1}\right)$ the problem (1.1) has at least one weak nontrivial solution.

Proof. Let

$$
\Omega=\{u \in X:\|u\| \leq \beta\}
$$

with $\beta \in(0,1)$.

For $u \in \Omega$, by (2.10), (2.12) and (2.13)

$$
\begin{aligned}
J_{\lambda}(u) & \geq \frac{\underline{\alpha}}{p^{+}} \sum_{k=1}^{T+1}|\Delta u(k-1)|^{p(k-1)}-\lambda\left(\overline{\frac{B_{2}}{r^{-}}} \sum_{k=1}^{T}|u(k)|^{r(k)}+\left|\underline{A_{2}}\right| \sum_{k=1}^{T}|u(k)|\right) \\
& \geq \frac{\underline{\alpha}}{p^{+}} T^{\frac{2-p^{+}}{2}}\|u\|^{p^{+}-\lambda}\left(\overline{\frac{B_{2}}{r^{-}}} \sum_{k=1}^{T}|u(k)|^{r^{+}}+\frac{\overline{B_{2}}}{r^{-}} \sum_{k=1}^{T}|u(k)|^{r^{-}}+\left|\underline{A_{2}}\right| \sqrt{T}\left(\sum_{k=1}^{T}|u(k)|^{2}\right)^{\frac{1}{2}}\right) \\
& \geq \frac{\underline{\alpha}}{p^{+}} T^{\frac{2-p^{+}}{2}}\|u\|^{p^{+}}-\lambda\left(\frac{\overline{B_{2}}}{r^{-}} c_{r^{+}}(T+1)\|u\|^{r^{+}}+\frac{\overline{B_{2}}}{r^{-}} c_{r^{-}}(T+1)\|u\|^{r^{-}}+\left|\underline{A_{2}}\right| \sqrt{T c_{2}}\|u\|\right) .
\end{aligned}
$$

For $u \in \partial \Omega$, we obtain

$$
J_{\lambda}(u) \geq \frac{\underline{\alpha}}{p^{+}} T^{-\frac{p^{+}-2}{2}} \beta^{p^{+}}-\lambda \beta\left(\frac{2 \overline{B_{2}}}{r^{-}}(T+1) \max \left(c_{r^{-}}, c_{r^{+}}\right)+\left|\underline{A_{2}}\right| \sqrt{T c_{2}}\right) .
$$

So for every $\lambda \in\left(0, \lambda_{1}\right)$

$$
J_{\lambda}(u)>0 \text { for all } u \in \partial \Omega
$$

with

$$
\lambda_{1}=\frac{\frac{\underline{\alpha}}{p^{+}} \beta^{\left(p^{+}-1\right)} T^{-\frac{p^{+}-2}{2}}}{\frac{2 \overline{B_{2}}}{r^{-}}(T+1) \max \left(c_{r^{-}}, c_{r^{+}}\right)+\left|\underline{A_{2}}\right| \sqrt{T c_{2}}} .
$$


Take $u \in X$ such that $u(k)>1$, for $k \in \mathbb{N}[1, T]$

$$
J_{\lambda}(u) \leq C_{1} \bar{\alpha} \sum_{k=1}^{T+1}\left(|\Delta u(k-1)|+\frac{|\Delta u(k-1)|^{p(k-1)}}{p^{-}}\right)-\lambda\left(-\left|\underline{A_{1}}\right| \sum_{k=1}^{T} u(k)+\frac{B_{1}}{r^{+}} \sum_{k=1}^{T}|u(k)|^{r^{-}}\right) .
$$

Let $u_{t} \in X$ defined in the following way $: u_{t}(k)=t$ for $k \in \mathbb{N}[1, T]$ and $u_{t}(0)=u_{t}(T+1)=0$.

For $t>1$, we have

$$
\begin{aligned}
J_{\lambda}\left(u_{t}\right) & \leq C_{1} \bar{\alpha}\left(2 t+\frac{t^{p(0)}+t^{p(T)}}{p^{-}}\right)-\lambda T\left(-\left|\underline{A_{1}}\right| t+\frac{\underline{B_{1}}}{r^{+}} t^{r^{-}}\right) \\
& \leq 4 C_{1} \bar{\alpha} t^{p^{+}}-\lambda T\left(-\left|\underline{A_{1}}\right| t+\frac{\underline{B_{1}}}{r^{+}} t^{r^{-}}\right)
\end{aligned}
$$

Since $r^{-}>p^{+}, \lim _{t \rightarrow+\infty} J_{\lambda}\left(u_{t}\right)=-\infty$; then there exists $t_{0}$ such that for $u_{t_{0}} \in X \backslash \Omega$

$$
J_{\lambda}\left(u_{t_{0}}\right)<\min _{u \in \partial \Omega} J_{\lambda}(u)
$$

$J_{\lambda} \in C^{1}(X, \mathbb{R})$, and according to Lemma 2.2 the problem (1.1) has at least one weak nontrivial solution.

\subsection{Case $p^{-}>r^{-}$.}

In this part we apply Ekeland's variational principle.

Proof. Take $\lambda \in\left(0, \lambda_{1}\right)$. In the proof of Theorem 3.2, for every $u \in \partial \Omega$, we have $J_{\lambda}(u)>0$.

Using Weierstrass theorem we obtain

$$
\inf _{u \in \partial \Omega} J_{\lambda}(u)>0
$$

Taking $u(k) \in(0, \beta)$, we have

$$
J_{\lambda}(u) \leq C_{1} \bar{\alpha} \sum_{k=1}^{T+1}\left(|\Delta u(k-1)|+\frac{|\Delta u(k-1)|^{p(k-1)}}{p^{-}}\right)-\lambda\left(-\left|\underline{A_{1}}\right| \sum_{k=1}^{T}|u(k)|+\frac{B_{1}}{r^{+}} \sum_{k=1}^{T}|u(k)|^{r(k)}\right) .
$$

For $t \in(0, \beta)$, assume that

$$
t<\left(p^{-r^{-}}\right) \sqrt{\frac{\lambda\left(-\max \left(\beta_{1}, \beta_{2}\right)\left|\underline{A_{1}}\right|+\frac{B_{1}}{r^{+}}\right)}{2 C_{1} \bar{\alpha}\left(\alpha_{1}+\frac{1}{p^{-}}\right)}} .
$$

We choose $k_{0} \in \mathbb{N}[1, T]$ such that $r\left(k_{0}\right)=r^{-}$.

Let $u_{0} \in X$ be a function such that $u_{0}\left(k_{0}\right)=t$ and $u_{0}(k)=0$ for any $k \in \mathbb{N}[1, T] \backslash\left\{k_{0}\right\}$. 
We obtain

$$
\begin{aligned}
J_{\lambda}\left(u_{0}\right) & \leq C_{1} \bar{\alpha}\left(2 t+\frac{t^{p\left(k_{0}-1\right)}+t^{p\left(k_{0}\right)}}{p^{-}}\right)-\lambda\left(-\underline{\underline{A_{1}}} \mid t+\frac{B_{1}}{\overline{r^{+}}} t^{r^{-}}\right) \\
& \leq 2 C_{1} \bar{\alpha}\left(t+\frac{t^{p^{-}}}{p^{-}}\right)-\lambda\left(-\left|\underline{A_{1}}\right| t+\frac{B_{1}}{r^{+}} t^{r^{-}}\right) .
\end{aligned}
$$

There exists $\alpha_{1}, \beta_{1} \geq 1$ such that $\alpha_{1} t^{p^{-}} \geq t$ and $\beta_{1} t^{r^{-}} \geq t$.

We have

$$
\begin{aligned}
J_{\lambda}\left(u_{0}\right) & \leq 2 C_{1} \bar{\alpha} t^{p^{-}}\left(\alpha_{1}+\frac{1}{p^{-}}\right)-\lambda\left(-\beta_{1}\left|\underline{A_{1}}\right|+\frac{B_{1}}{\underline{r^{+}}}\right) t^{r^{-}} \\
& \leq 2 C_{1} \bar{\alpha} t^{p^{-}}\left(\alpha_{1}+\frac{1}{p^{-}}\right)-\lambda\left(-\max \left(\beta_{1}, \beta_{2}\right)\left|\underline{A_{1}}\right|+\frac{B_{1}}{r^{+}}\right) t^{r^{-}}<0 .
\end{aligned}
$$

Thus, $J_{\lambda}\left(u_{0}\right)<0$ for $u_{0} \in \operatorname{Int}(\Omega)$.

Therefore,

$$
-\infty<\inf _{u \in \operatorname{Int}(\Omega)} J_{\lambda}(u)<0
$$

So,we have

$$
\inf _{u \in \operatorname{Int}(\Omega)} J_{\lambda}(u)<\inf _{u \in \partial \Omega} J_{\lambda}(u) .
$$

Using the proof of [14] we have

$$
0<\varepsilon<\inf _{u \in \partial \Omega} J_{\lambda}(u)-\inf _{u \in \operatorname{Int}(\Omega)} J_{\lambda}(u) .
$$

Applying Ekeland's variationnal principle to the functional $J_{\lambda}: \Omega \longrightarrow \mathbb{R}$ we find $u_{\varepsilon} \in \Omega$ such that

$$
\begin{aligned}
J_{\lambda}\left(u_{\varepsilon}\right) & <\inf _{u \in \Omega} J_{\lambda}(u)+\varepsilon \\
& <J_{\lambda}(u)+\varepsilon\left\|u-u_{\varepsilon}\right\| \quad \text { for } \quad u \neq u_{\varepsilon} .
\end{aligned}
$$

Since

$$
J_{\lambda}\left(u_{\varepsilon}\right)<\inf _{u \in \Omega} J_{\lambda}(u)+\varepsilon \leq \inf _{u \in \operatorname{Int}(\Omega)} J_{\lambda}(u)+\varepsilon<\inf _{u \in \partial \Omega} J_{\lambda}(u),
$$

we deduce $u_{\varepsilon} \in \operatorname{Int}(\Omega)$.

Now, we define $L_{\lambda}: \Omega \longrightarrow \mathbb{R}$ by

$$
L_{\lambda}(u)=J_{\lambda}(u)+\varepsilon\left\|u-u_{\varepsilon}\right\| \quad \text { for } \quad u \neq u_{\varepsilon} .
$$

We have $u_{\varepsilon}$ as a minimum of $L_{\lambda}$ and therefore

$$
\frac{L_{\lambda}\left(u_{\varepsilon}+t v\right)-L_{\lambda}\left(u_{\varepsilon}\right)}{t}+\varepsilon\|v\| \geq 0
$$


for any $v \in \Omega$ and a small enough positive $t$.

We deduce that

$$
\frac{J_{\lambda}\left(u_{\varepsilon}+t v\right)-J_{\lambda}\left(u_{\varepsilon}\right)}{t}+\varepsilon\|v\| \geq 0
$$

Letting $t \rightarrow 0$, it follows that

$$
\left\langle J_{\lambda}^{\prime}\left(u_{\varepsilon}\right), v\right\rangle+\varepsilon\|v\|>0
$$

and we obtain

$$
\left\|J_{\lambda}^{\prime}\left(u_{\varepsilon}\right)\right\| \leq \varepsilon .
$$

There exists a sequence $\left\{z_{n}\right\} \subset \operatorname{Int}(\Omega)$ such that

$$
J_{\lambda}\left(z_{n}\right) \rightarrow \inf _{u \in \Omega} J_{\lambda}(u) \quad \text { and } \quad J_{\lambda}^{\prime}\left(z_{n}\right) \rightarrow 0
$$

Since $\left\{z_{n}\right\}$ is bounded in $X$ there exists $z_{0} \in X$ such that, up to a subsequence, $\left\{z_{n}\right\}$ converges to $z_{0} \in X$. Thus

$$
J_{\lambda}\left(z_{0}\right)=\inf _{u \in \Omega} J_{\lambda}(u) \quad \text { and } \quad J_{\lambda}^{\prime}\left(z_{0}\right)=0 .
$$

$z_{0}$ is one weak nontrivial solution for problem (1.1).

\subsection{Multiple solutions.}

In this section we prove the existence of at least two weak nontrivial solutions of the problem (1.1).

Theorem 3.3. Assume that (2.4), (2.7) holds. Let $r^{-}>p^{+}$and $\delta>1$.

For any $\lambda \in\left(0, \frac{\underline{\alpha}\left(C_{2} \delta^{p^{-}}-C_{3}\right)+\sigma_{1} \delta^{2}}{\delta^{r^{+}}\left(\underline{\left|A_{2}\right|} \mid \sqrt{T c_{2}}+2 \max \left(c_{r^{+}}, c_{r^{-}}\right) \bar{B}_{2}(T+1)\right)}\right)$ the problem (1.1) has at least two weak nontrivial solutions where one solution satisfies $\|u\|>1$.

Proof. Let

$$
\Omega_{\delta}:=\{u \in X:\|u\| \leq \delta\} ; \quad \Omega_{\nu}:=\{u \in X:\|u\| \geq \nu\},
$$

where $\nu \in(1, \delta)$.

Assume that $u_{0} \in X$ is a local minimizer of $J_{\lambda}$ in $\Omega=\Omega_{\delta} \cap \Omega_{\nu}$.

If $u_{0} \in \operatorname{Int}(\Omega)$ by using Lemma 2.2 we get $J_{\lambda}\left(u_{0}\right)<\min _{u \in \partial \Omega_{\delta}} J_{\lambda}(u)$.

Now suppose that $u_{0} \in \partial \Omega_{\delta}$, by Theorem 2.3 there exist $\sigma_{0}, \sigma_{1}, \sigma_{2} \geq 0$ with $\sigma_{0}^{2}+\sigma_{1}^{2}+\sigma_{2}^{2}>0$ such that for all $v \in X$

$$
\sigma_{1}\left(\left\|u_{0}\right\|^{2}-\delta^{2}\right)=0 \quad \text { and } \quad \sigma_{2}\left(\nu^{2}-\left\|u_{0}\right\|^{2}\right)=0
$$


and

$$
\sigma_{0}\left\langle J_{\lambda}^{\prime}\left(u_{0}\right), v\right\rangle+\sigma_{1}\left\langle u_{0}, v\right\rangle-\sigma_{2}\left\langle u_{0}, v\right\rangle=0
$$

Since $u_{0} \in \partial \Omega_{\delta}$, we have $\left\|u_{0}\right\|=\delta$ and $\sigma_{2}=0$.

Taking $v=u_{0}$ and $\sigma_{0}=1$ we see that

$$
\sum_{k=1}^{T+1} \alpha(k-1) a\left(k-1, \Delta u_{0}(k-1)\right) \Delta u_{0}(k-1)+\sigma_{1}\left\|u_{0}\right\|^{2}=\lambda \sum_{k=1}^{T} f\left(k, u_{0}(k)\right) u_{0}(k) .
$$

By (2.4) and (2.11)

$$
\sum_{k=1}^{T+1} \alpha(k-1) a\left(k-1, \Delta u_{0}(k-1)\right) \Delta u_{0}(k-1)+\sigma_{1}\left\|u_{0}\right\|^{2} \geq \underline{\alpha}\left(C_{2} \delta^{p^{-}}-C_{3}\right)+\sigma_{1} \delta^{2}
$$

Using (2.7), (2.10) and (2.13)

$$
\begin{aligned}
& \lambda \sum_{k=1}^{T} f\left(k, u_{0}(k)\right) u_{0}(k) \\
\leq & \lambda\left(\left|\underline{\mid A_{2}}\right| \sum_{k=1}^{T}\left|u_{0}(k)\right|+\overline{B_{2}} \sum_{k=1}^{T}\left|u_{0}(k)\right|^{r^{+}}+\overline{B_{2}} \sum_{k=1}^{T}\left|u_{0}(k)\right|^{r^{-}}\right) \\
\leq & \lambda\left(\left|\underline{\mid A_{2}}\right| \sqrt{T} \sqrt{\sum_{k=1}^{T}\left|u_{0}(k)\right|^{2}}+c_{r^{+}} \overline{B_{2}} \sum_{k=1}^{T+1}\left|\Delta u_{0}(k)\right|^{r^{+}}+c_{r^{-}} \overline{B_{2}} \sum_{k=1}^{T+1}\left|\Delta u_{0}(k)\right|^{r^{-}}\right) \\
\leq & \lambda\left(\left|\underline{\mid A_{2}}\right| \sqrt{T c_{2}}|| u_{0}||+c_{r^{+}} \overline{B_{2}}(T+1)\left\|u_{0}\right\|^{r^{+}}+c_{r^{-}} \overline{B_{2}}(T+1)\left\|u_{0}\right\|^{r^{-}}\right) \\
\leq & \lambda \delta^{r^{+}}\left(\underline{\mid A_{2}} \mid \sqrt{T c_{2}}+2 \max \left(c_{r^{+}}, c_{r^{-}}\right) \overline{B_{2}}(T+1)\right) .
\end{aligned}
$$

So

$$
\underline{\alpha}\left(C_{2} \delta^{p^{-}}-C_{3}\right)+\sigma_{1} \delta^{2} \leq \lambda \delta^{r^{+}}\left(\left|\underline{A_{2}}\right| \sqrt{T c_{2}}+2 \max \left(c_{r^{+}}, c_{r^{-}}\right) \overline{B_{2}}(T+1)\right)
$$

This is contradictory.

Hence $u_{0} \in \operatorname{Int}(\Omega)$ is a nontrivial minimizer of $J_{\lambda}$.

In the proof of Theorem (3.2) we show that there exists $u_{1} \in X \backslash \Omega$ such that $J_{\lambda}\left(u_{1}\right)<$ $\min _{u \in \partial \Omega_{\delta}} J_{\lambda}(u)$

According to Lemma 2.2, there exists $u^{*} \in X$ such that $u^{*}$ is a critical point of $J_{\lambda}$.

Finally, $u_{0}$ and $u^{*}$ are two different weak nontrivial solutions to the problem (1.1) and since $u_{0} \in \operatorname{Int}(\Omega)$, it is easy to see that $\left\|u_{0}\right\|>1$. 


\section{An extension}

In this section, we show that the existence result obtained in (1.1) can be extended to more general discrete boundary value problems of the form

$$
\left\{\begin{array}{l}
-\Delta[\alpha(k-1) a(k-1, \Delta u(k-1))]+|u|^{q(k)-2} u(k)=\lambda f(k, u(k)), k \in \mathbb{N}[1, T], \\
u(0)=u(T+1)=0
\end{array}\right.
$$

with $q: \mathbb{N}[1, T] \longrightarrow(2,+\infty)$.

A function $u \in X$ is a solution of problem (4.1) if for any $v \in X$,

$\sum_{k=1}^{T+1} \alpha(k-1) a(k-1, \Delta u(k-1)) \Delta v(k-1)+\sum_{k=1}^{T}|u(k)|^{q(k)-2} u(k) v(k)-\lambda \sum_{k=1}^{T} f(k, u(k)) v(k)=0$

Theorem 4.1. Assume that (2.5) and (2.7) hold. For all $p^{-}>r^{+}$there exists $\lambda_{2} \in(0,+\infty)$ such that for all $\lambda>\lambda_{2}$ the problem (4.1) has at least one weak nontrivial solution.

Proof. In this part we use the proof of Theorem 3.1. For $u \in X$, we define the energy functional $J_{\lambda}$ by

$$
J_{\lambda}(u)=\sum_{k=1}^{T+1} \alpha(k-1) A(k-1, \Delta u(k-1))+\sum_{k=1}^{T} \frac{1}{q(k)}|u|^{q(k)}-\lambda \sum_{k=1}^{T} F(k, u(k)) .
$$

The functional $J_{\lambda}$ is well defined, weakly lower semi continuous and is of class $C^{1}(X, \mathbb{R})$ with a derivative given by

$\left\langle J_{\lambda}^{\prime}(u), v\right\rangle=\sum_{k=1}^{T+1} \alpha(k-1) a(k-1, \Delta u(k-1)) \Delta v(k-1)+\sum_{k=1}^{T}|u|^{q(k)-2} u(k) v(k)-\lambda \sum_{k=1}^{T} f(k, u(k)) v(k)$,

for all $u, v \in X$.

Since

$$
\sum_{k=1}^{T} \frac{1}{q(k)}|u|^{q(k)} \geq 0
$$

we have

$$
J_{\lambda}(u) \geq \sum_{k=1}^{T+1} \alpha(k-1) A(k-1, \Delta u(k-1))-\lambda \sum_{k=1}^{T} F(k, u(k)) .
$$

According to Proposition 3.1 we deduce that the functional $J_{\lambda}$ is coercive.

Let $u_{\lambda}$ be a global minimizer of $J_{\lambda}$, taking $u_{0}$ such that $u_{0}\left(k_{0}\right)=t_{0}$ and $u_{0}(k)=0$, for 
$k \in \mathbb{N}[0, T+1] \backslash\left\{k_{0}\right\}$ where $t_{0}>1$ is a fixed real, we have

$$
J_{\lambda}\left(u_{0}\right) \leq 4 C_{1} \bar{\alpha} t_{0}^{p^{+}}+\frac{t_{0}^{q^{+}}}{q^{-}}-\lambda t_{0}\left(-\max \left(\beta_{1}, \beta_{2}\right)\left|\underline{A_{1}}\right|+\frac{B_{1}}{r^{+}}\right),
$$

where

$$
\lambda_{2}=\frac{4 C_{1} \bar{\alpha} t_{0}^{p^{+}-1}+\frac{t_{0}^{q^{+}-1}}{q^{-}}}{-\max \left(\beta_{1}, \beta_{2}\right)\left|\underline{A_{1}}\right|+\frac{B_{1}}{r^{+}}} .
$$

It follows that $J_{\lambda}\left(u_{\lambda}\right)<0$ for any $\lambda>\lambda_{2}$. $u_{\lambda}$ is one weak nontrivial solution of problem (4.1).

Lemma 4.1. Assume that (2.5), (2.7) hold and $r^{-}>\max \left(p^{+}, q^{+}\right)$. Then for any $\lambda>0$ the functional $J_{\lambda}$ satisfies the Palais-Smale condition.

Proof. By mimicking the proof of Lemma 3.1, we obtain

$$
\begin{aligned}
J_{\lambda}\left(u_{n}\right) \leq & C_{1} \bar{\alpha}\left(\sqrt{T+1}\left\|u_{n}\right\|+\frac{(T+1)\left\|u_{n}\right\|^{p^{+}}}{p^{-}}+\frac{T+1}{p^{-}}\right)+\sum_{k=1}^{T} \frac{1}{q(k)}\left|u_{n}(k)\right|^{q(k)} \\
& -\lambda\left(-\underline{\underline{A_{1}} \mid} \sqrt{T} c_{2}\left\|u_{n}\right\|^{2}+\frac{\left.2^{-r^{-}(T+1)^{\frac{2-r^{-}}{2}} \underline{B_{1}}}\left\|u_{n}\right\|^{r^{-}}\right)}{r^{+}}\right. \\
\leq & C_{1} \bar{\alpha}\left(\sqrt{T+1}\left\|u_{n}\right\|+\frac{(T+1)\left\|u_{n}\right\|^{p^{+}}}{p^{-}}+\frac{T+1}{p^{-}}\right)+\frac{c_{q^{+}}(T+1)\left\|u_{n}\right\|^{q^{+}}}{q^{-}} \\
& -\lambda\left(-\left|\underline{A_{1}}\right| \sqrt{T} c_{2}\left\|u_{n}\right\|^{2}+\frac{2^{-r^{-}}(T+1)^{\frac{2-r^{-}}{2}} \underline{B_{1}}}{r^{+}}\left\|u_{n}\right\|^{r^{-}}\right) .
\end{aligned}
$$

Since $r^{-}>\max \left(p^{+}, q^{+}\right)$then the sequence $\left\{u_{n}\right\}$ is bounded.

In what follow, we apply the mountain pass geometry Lemma.

Theorem 4.2. Let $r^{-}>\max \left(p^{+}, q^{+}\right)$and the condition (2.7) holds. Then for $\lambda \in\left(0, \lambda_{1}\right)$, the problem (4.1) has at least one weak nontrivial solution.

Proof. We will refer to the proof of theorem 3.2.

Let

$$
\Omega=\{u \in X:\|u\| \leq \beta\}
$$

with $\beta \in(0,1)$. 
For $u \in \Omega$

$$
J_{\lambda}(u) \geq \frac{\underline{\alpha}}{p^{+}} \sum_{k=1}^{T+1}|\Delta u(k-1)|^{p(k-1)}-\lambda\left(\overline{\frac{B_{2}}{r^{-}}} \sum_{k=1}^{T}|u(k)|^{r(k)}+\left|\underline{A_{2}}\right| \sum_{k=1}^{T}|u(k)|\right) .
$$

For all $\lambda \in\left(0, \lambda_{1}\right)$ we obtain $\inf _{u \in \partial \Omega} J_{\lambda}(u)>0$.

Let $u_{t} \in X$ be defined as follows : $u_{t}(k)=t$ for $k \in \mathbb{N}[1, T]$ and $u_{t}(0)=u_{t}(T+1)=0$.

For $t>1$ we have

$$
J_{\lambda}(u) \leq 4 C_{1} \bar{\alpha} t^{p^{+}}+T t^{q^{+}}-\lambda T\left(-\left|\underline{A_{1}}\right| t+\frac{\underline{B_{1}}}{r^{+}} t^{r^{-}}\right) .
$$

Since $r^{-}>\max \left(p^{+}, q^{+}\right), \lim _{t \rightarrow+\infty} J_{\lambda}\left(u_{t}\right)=-\infty$; then it exist $t_{1}$ such that

$$
u_{t_{1}} \in X \backslash \Omega \quad J_{\lambda}\left(u_{t_{1}}\right)<\min _{u \in \partial \Omega} J_{\lambda}(u) .
$$

The functional $J_{\lambda} \in C^{1}(X, \mathbb{R})$, and the assumptions of Lemma 2.2 are verified, then the problem (4.1) has at least one weak nontrivial solution.

We apply Ekeland's variational principle with $\min \left(p^{-}, q^{-}\right)>r^{-}$; we will use the result of case $p^{-}>r^{-}$.

For $\lambda \in\left(0, \lambda_{1}\right)$

$$
\inf _{u \in \partial \Omega} J_{\lambda}(u)>0
$$

For $t \in(0, \beta)$, assume that

$$
t<\left(\min \left(p^{-}, q^{-}\right)-r^{-}\right) \sqrt{\frac{\lambda\left(-\max \left(\beta_{1}, \beta_{2}\right)\left|\underline{A_{1}}\right|+\frac{B_{1}}{r^{+}}\right)}{2 C_{1} \bar{\alpha}\left(\alpha_{2}+\frac{1}{p^{-}}+\frac{1}{2 C_{1} \bar{\alpha} q^{-}}\right)}}
$$

we choose $k_{0} \in \mathbb{N}[1, T]$ such that $r\left(k_{0}\right)=r^{-}$.

Let $u_{0} \in X$ be a function such that $u_{0}\left(k_{0}\right)=t$ and $u_{0}(k)=0$, for any $k \in \mathbb{N}[1, T] \backslash\left\{k_{0}\right\}$.

We obtain

$$
J_{\lambda}\left(u_{0}\right) \leq 2 C_{1} \bar{\alpha}\left(t+\frac{t^{p^{-}}}{p^{-}}\right)+\frac{t^{q^{-}}}{q^{-}}-\lambda\left(-\underline{\mid A_{1}} \mid t+\frac{B_{1}}{r^{+}} t^{r^{-}}\right) .
$$

There exists $\alpha_{2}, \beta_{2}>1$ such that $\alpha_{2} t^{\min \left(p^{-}, q^{-}\right)} \geq t$ and $\beta_{2} t^{r^{-}}>t$.

We have

$$
J_{\lambda}\left(u_{0}\right) \leq 2 C_{1} \bar{\alpha} t^{\min \left(p^{-}, q^{-}\right)}\left(\alpha_{2}+\frac{1}{p^{-}}+\frac{1}{2 C_{1} \bar{\alpha} q^{-}}\right)-\lambda\left(-\max \left(\beta_{1}, \beta_{2}\right)\left|\underline{A_{1}}\right|+\frac{B_{1}}{\overline{r^{+}}}\right) t^{r^{-}}<0 .
$$

Thus, $J_{\lambda}\left(u_{0}\right)<0$ for $u_{0} \in \operatorname{Int}(\Omega)$.

By the same reasoning we prove that the problem (4.1) has at least one weak nontrivial 
solution.

Now we will prove that problem (4.1) has at least two weak nontrivial solution.

In the case of multiple solutions we will use the Theorem 3.3 and the same sets defined previously.

Indeed, let $u_{0} \in X$ a local minimizer of $J_{\lambda}$ on $\Omega=\Omega_{\delta} \cap \Omega_{\nu}$. If $u_{0} \in \operatorname{Int}(\Omega)$ we have $J_{\lambda}\left(u_{0}\right)<\min _{u \in \partial \Omega_{\delta}} J_{\lambda}(u)$.

Assume that $u_{0} \in \partial \Omega_{\delta}$, we have $\sigma_{2}=0$, taking $v=u_{0}$ and $\sigma_{0}=1$ we get

$$
\sum_{k=1}^{T+1} \alpha(k-1) a\left(k-1, \Delta u_{0}(k-1)\right) \Delta u_{0}(k-1)+\sum_{k=1}^{T}\left|u_{0}\right|^{q(k)}+\sigma_{1}\left\|u_{0}\right\|^{2}=\lambda \sum_{k=1}^{T} f\left(k, u_{0}(k)\right) u_{0}(k) .
$$

By the same reasoning we prove that problem (4.1) has at least two weak nontrivial solutions.

\section{ACKNOWLEDGMENT}

The authors express their deepest thanks to the editor and anonymous referee for their comments and suggestions on the article.

\section{REFERENCES}

[1] R.P. Argawal; Difference Equations and Inequalities, Marcel Dekker, New York, 1992.

[2] J.M. Borwein, A.S. Lewis; Convex analysis and nonlinear optimization. Theory and examples, 2nd ed., CMS Books in Mathematics/Ouvrages de Mathématiques de la SMC 3. New York, NY: Springer. Xii, 2006.

[3] X. Cai and J. Yu; Existence theorems for second-order discrete boundary value problems, J. Math. Anal. Appl. 320 (2006), 649-661.

[4] G. Dajun, Nonlinear Functional Analysis, Shandong Science and Technology Press, 1985.

[5] S.N. Elaydi; An Introduction to Difference Equations, Undergraduate Texts in Mathematics, Springer-Verlag, New York, 1999.

[6] DG. De Figueiredo; Lectures on the Ekeland Variational Principe with Applications and Detours, Bombay: Tata Institute of Fundamental Research, 1989.

[7] M. Galewski and R. Wieteska; Existence and multiplicity of positive solutions for discrete anisotropic equations , Turk. J. Math. 38 (2014), 297-310.

[8] M. Galewski, R. Wieteska, On the system of anisotropic discrete BVPs, J. Differ. Equ. Appl. 19 (2013), 1065-1081.

[9] A. Guiro, I. Nyanquini, S. Ouaro, On the solvability of discrete nonlinear Neumann problems involving the p(x)-Laplacian, Adv. Diff. Equ. 2011 (2011), 32.

[10] I. Ibrango, R. Sanou, B. Koné, A. Guiro, Weak homoclinic solutions of anisotropic discrete nonlinear system with variable exponent, Nonauton. Dyn. Syst. 7 (2020), 22-31. 
[11] B. Koné and S. Ouaro; Weak solutions for anisotropic discrete boundary value problems. J. Differ. Equ. Appl. 16(2) (2010), 1-11.

[12] V. Lakshmikantham and D. Trigiante; Theory of Difference Equations: Numerical Methods and Applications, Academic Press, New York, 1988.

[13] J. Mawhin; Problèmes de Dirichlet variationnels non linéaires, Les Presses de l’Université de Montréal, 1987.

[14] M. Mihailescu, V. Radulescu and S. Tersian; Eigenvalue problems for anisotropic discrete boundary value problems, J. Differ. Equ. Appl. 15 (2009), 557-567.

[15] R. Sanou, I. Ibrango, B. Koné, A. Guiro, Weak solutions to Neumann discrete nonlinear system of Kirchhoff type, Cubo. 21 (2019), 75-91. 